\title{
Standard Grey Potential and Its Application in Grey Target Decision
}

\author{
Aiqing Ruan ${ }^{1}$, Xiaoyong Yin $^{2, *}$, Qiu Chen ${ }^{3}$ and Yinao Wang ${ }^{4}$ \\ ${ }^{1,2}$ School of Economics and Management, Dongguan University of Technology, Dongguan, Guangdong Province, 523808, P.R. \\ China \\ ${ }^{3}$ Wenzhou Administrative College, Wenzhou, Zhejiang Province, 325035, P.R. China \\ ${ }^{4}$ College of Mathematics and Information Science, Wenzhou University, Wenzhou, Zhejiang Province, 325035, P.R. China \\ ${ }^{*}$ Corresponding author
}

\begin{abstract}
Grey target decision-making is one of important problems of decision-making theory. It is critical to express uncertain information effectively and depose them in reasonable and simple way. Firstly this paper makes a brief overview of the existing method for Grey target decision. Then the conception of Grey potential degree system is introduced and the conception of standard Grey Potential Degree is build up in the paper. And a new Grey potential-based method on Grey target clustering is proposed. At the same time the standard Grey potential and its application in multiple criteria is studied. It is presented by three examples, that the Grey potential-based method could be used to solve the problem of Grey target decision conveniently and effectively.
\end{abstract}

Keywords-grey target; decision-making; grey number; grey potential degree

\section{INTRODUCTION}

In traditional decision-making theory, the parameters of model are usually of determined values. However in real life, because of objective conditions and human cognition limitation, it is very hard to obtain complete information; hence uncertainty theory becomes an important branch of decisionmaking problem.

Grey systems theory is another method to do with uncertain decision-making problems. Grey number is used to describe uncertain information in the theory. Deng is the pioneer of Grey systems theory who first used described uncertain problems with Grey numbers [1].

The thought of Gray target [2] is, for a set of patterns in the sequence, to find the data which is nearest to target value and construct a standard mode, each mode and standard mode constitutes gray target, the standard mode is the bull's-eye. Grey Target is a method for processing multiple program evaluation and optimization of multiple objective problem. The earliest introduction of Interval Number into Grey Target Decision Model is Yaoguo Dang, Sifeng Liu and Bin Liu's research. Important research Grey Target include information theory established Shannon, using entropy to measure the amount of information; Chanyao Gu and Wanhua Qiu[3] made definition of entropy and introduced into decision-making; Entropy is used by Mon et al. To decide weight[4]; Wang et al study using objective programming, quadratic programming, and dynamic programming model to determine the index weight [5]. Sifeng Liu [6], Yaoguo Dang [7], Yongming Chen
[8] all studied on Grey Target decision-making. Grey Target has also been applied and Evaluation. To increase the amount of information to improve decision-making accuracy, Guangzhi $\mathrm{Bu}$ built up fuzzy comprehensive evaluation of three parameters interval numbers [9]. In addition Qizhou Hu [10], Xinfang Wang [11], Rong Lan [12], Luo Dang [13] and Jie Song [14] studied and explored three-parameter interval gray numbers.

To compare Grey number is very important to obtain scientific and reasonable results for a Grey target decisionmaking problem. But there is very little study on comparing or ranking Grey numbers. Xie and Liu, in paper [15], put forward novel methods on comparing Grey number, of which the probability density function is used. In Zeng's research on Grey target decision-making model, the information distribution of Grey number is asked to know [16].

However, in the actual application of Grey number, there are some problems. (1) It is difficult to know the probability density function or the whiten function of Grey number. (2) If Grey number is compared and deposed through whiten value which is most commonly used, much information of Grey number will be lost, at the same time the value of Grey number in uncertainty is lost partly. (3) In normal condition, it is hard to supply additional information for decision-making.

So that, this paper focuses on handling directly Grey number without whiten value and without distribution function in Grey target decision-making. Furthermore, approach of Grey potential degree could treat the data with both comparing and standardization work. Grey potential could be used as a form of metrics in Grey target decision-making.

In this paper, we will propose the conception of Grey potential degree and discuss its application in Grey target decision. The remaining paper is organized as follows. In Section II, the conception system of Grey number and Grey potential degree is put forward. And the structure of Grey potential degree is presented and especially Standard Grey Target and Standard Grey potential degree. In Section III, the application of standard Grey potential degree, the model of Basic Grey Target Clustering Model is studied. Finally, the typical numerical example are presented to demonstrate the effectiveness of the method in Section IV. Section V comes to the conclusions. 


\section{CONCEPTIONS OF GREy Potential DegReE}

\section{A. Grey Potential Degrees}

Definition 2.1 (Grey number) because of the defect of information due to various reasons, the exact value of the characteristics cannot be determined but the scope of exact value can be decided, so the description of the characteristics can use Grey number $\otimes_{i}$, denoted as $\otimes_{i} \in\left[a_{i j}, b_{i j}\right], \quad a_{i j} \neq b_{i j}$.

Definition 2.2 (Grey number whitening and true value) for Grey number $\otimes_{i}$, when more and more information is provided, the scope of Grey number $\otimes_{i}$ becomes smaller and smaller, this process is called Grey number whitening. When complete information is supplied, the Grey number whitening is finished and true value $d_{i}^{*}$ of characteristics description $\otimes_{i}$ is determined.

When no further information is provided and Grey number has to be deposed without any whitening. So Zhigeng Fang put forward the concept of Grey potential degree to build a comparative framework for the interval Grey number in his doctoral thesis [17]. The improved conceptions of Grey potential degree are put forward in the past studies [18,19] and are further developed here.

Definition 2.3 (Grey Superiority Potential Degree of Grey Numbers $i-t o-j, G S P D_{i \rightarrow j}$ in short) for any two Grey numbers $\otimes_{i} \in\left[a_{i}, b_{i}\right], \quad a_{i} \neq b_{i}$ and $\otimes_{j} \in\left[a_{j}, b_{j}\right], b_{i}-b_{j}$ is called Grey superior dominance. $\frac{b_{i}-b_{j}}{b_{i}-a_{i}}$ is called Grey Superiority Potential Degree of Grey Number $\otimes_{i}$ to Grey number $\otimes_{j}$, denoted as $G S P D_{i \rightarrow j}$.

Definition 2.4 (Grey Inferior Potential Degree of Grey Numbers $i-t o-j, G I P D_{i \rightarrow j}$ in short) for any two Grey numbers $\otimes_{i} \in\left[a_{i}, b_{i}\right], \quad a_{i} \neq b_{i}$ and $\otimes_{j} \in\left[a_{j}, b_{j}\right]$, $a_{i}-a_{j}$ is called Grey inferior dominance. $\frac{a_{i}-a_{j}}{b_{i}-a_{i}}$ is called Grey Inferior Potential Degree of Grey Number $\otimes_{i}$ to Grey number $\otimes_{j}$, denoted as $G I P D_{i \rightarrow j}$.

The realistic condition of Grey numbers are more complex, only $G S P D_{i \rightarrow j}$ or $G I P D_{i \rightarrow j}$ is not enough for full description of the relationship between the Grey numbers $\otimes_{i}$ and $\otimes_{j}$. Therefore $G S P D_{i \rightarrow j}$ and $G I P D_{i \rightarrow j}$ need to be combined.

At the same time, Grey Superiority Potential Degree of Grey Numbers focus on superiority potential and Grey Inferior Potential Degree focus on inferior potential. Superiority potential means good and positive potential and inferior potential means bad and negative possibility. In real situation, the decision-maker has different focus in different situation. Therefore, Grey Total Potential Degree of Grey Numbers is defined as following way.
Definition 2.5 (Grey Total Potential Degree of Grey Numbers $\otimes_{i}$ to $\otimes_{j}, G T P D_{i \rightarrow j}$ in short) For any two Grey numbers $\otimes_{i} \in\left[a_{i}, b_{i}\right], a_{i} \neq b_{i}$, and $\otimes_{j} \in\left[a_{j}, b_{j}\right]$, the sum of Grey Superiority Potential Degree and Grey Inferior Potential Degree of Grey number $\otimes_{i}$ to Grey number $\otimes_{j}$, is called Grey Total Potential Degree of Grey number $\otimes_{i}$ to Grey number $\otimes_{j}$, denoted as $G T P D_{i \rightarrow j}$, that is, $G T P D_{i \rightarrow j}=\alpha \cdot G S P D_{i \rightarrow j}+\beta \cdot G I P D_{i \rightarrow j}, \quad \alpha+\beta=1, \alpha, \beta \in[0,1]$. Here, $\alpha$ is Superiority coefficient, and $\beta$ is called Inferior coefficient. The value of these two coefficients displays the proportional relationship between Superiority Potential Degree which is representative of advantage potential, and Grey Inferior Potential Degree which is on behalf of risk, when the issue is considered in integrated way

Here $G T P D_{i \rightarrow j}$ is the sum of $G S P D_{i \rightarrow j}$ and $G I P D_{i \rightarrow j}$, and it can be used to compare two Grey numbers. In order to convenient to compare the Grey numbers by Grey Total Potential Degree, as follows give judging rules.

Definition 2.6 the Judgment Rules of Grey Total Potential Degree of Grey Numbers $\otimes_{i}$ to $\otimes_{j}$ are shown as follows.

For any two Grey numbers $\otimes_{i} \in\left[a_{i}, b_{i}\right], a_{i} \neq b_{i}$, and $\otimes_{j} \in\left[a_{j}, b_{j}\right]$,

1) When $G T P D_{i \rightarrow j}=0$, Grey numbers $\otimes_{i}$ and $\otimes_{j}$ have the Equal Grey Total Potential Degree, expressed as $\otimes_{i} \stackrel{p}{=} \otimes_{j}$.

2) When $G T P D_{i \rightarrow j} \succ 0$, Grey Number $\otimes_{i}$ has Positive Grey Total Potential Degree to Grey number $\otimes_{j}$, denoted as $\otimes_{i} \stackrel{p}{\succ}_{j}$. And $\otimes_{i}$ is Superior Potential Grey number, $\otimes_{j}$ is Inferior Potential Grey number.

3) When $G T P D_{i \rightarrow j} \prec 0$, Grey Number $\otimes_{i}$ has Negative Grey Total Potential Degree to Grey number $\otimes_{j}$, denoted as $\otimes_{i} \stackrel{p}{\prec} \otimes_{j}$. And $\otimes_{i}$ is Inferior Potential Grey number, $\otimes_{j}$ is Superior Potential Grey number.

There are some characters about Grey potential degree.

Theorem 2.1 for any three interval Grey numbers, $\otimes_{i} \in\left[a_{i}, b_{i}\right]\left(a_{i} \neq b_{i}\right), \otimes_{j} \in\left[a_{j}, b_{j}\right]\left(a_{j} \neq b_{j}\right)$, and $\otimes_{k} \in\left[a_{k}, b_{k}\right]$, if $\otimes_{i} \stackrel{p}{\prec} \otimes_{j}$ and $\otimes_{j} \stackrel{p}{\prec} \otimes_{k}$ exit, there will be $\otimes_{i} \prec \otimes_{k}$.

Theorem 2.2 for any four interval Grey numbers,

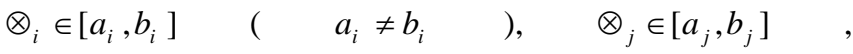


$\otimes_{s} \in\left[a_{s}, b_{s}\right] \otimes_{s} \in\left[a_{s}, b_{s}\right]\left(a_{s} \neq b_{s}\right)$ and $\otimes_{t} \in\left[a_{t}, b_{t}\right]$, if $\otimes_{i} \stackrel{p}{\prec} \otimes_{j}$ and $\otimes_{s} \stackrel{p}{\prec} \otimes_{t}$ exit, there will be $\otimes_{i}+\otimes_{s} \stackrel{p}{\prec} \otimes_{j}+\otimes_{t}$.

The values of Grey Potential Degrees tell us the relationship between Grey numbers. Therefore the distribution of the values of Grey Potential Degrees should be discussed further in order to compare Grey numbers.

\section{B. Standard Grey Target and Standard Grey potential degree}

The template is used to format your paper and style the text. All margins, column widths, line spaces, and text fonts are prescribed; please do not alter them. You may note peculiarities. For example, the head margin in this template measures proportionately more than is customary. This measurement and others are deliberate, using specifications that anticipate your paper as one part of the entire proceedings, and not as an independent document. Please do not revise any of the current designations.

In order to compare alternatives, consistent standard should be built up. In Grey target decision problem, there are two levels of standard. The alternatives should be selected by values of indexes. So different indexes should be compared and integrated. For each index, which maybe have different source or evaluated by many experts, it needs to combine different information by consistent standard.

It is considered that a standard Grey target is set for each index. And then compared to standard Grey target, the Grey potential degree could be used to compare and integrate.

Assume that there is evaluation Grey matrix $M(\otimes)=\left(\otimes_{i j}\right)_{m \times n}, \otimes_{i j} \in\left[a_{i j}, b_{i j}\right], \quad a_{i j} \neq b_{i j}, \quad i=1, \cdots, m$, $j=1, \cdots, n$. $\otimes_{i j}$ is the Grey evaluation of Object-i on Index-j, or $\otimes_{i j}$ is the Grey evaluation of Object-i by Expert-j, $\otimes_{i j}$ is the Grey evaluation of Object-i by or Source-j which may be judged by different ways. How to set standard Grey target?

Because different index has different measurement scale, each standard Grey target, denoted as $\otimes_{s}$ should be set for each index-j according to the character of index. However the most important thing is that if every standard Grey target $\otimes_{s}$ for every index follows the same principle and their Grey potential degrees are calculated by the same approach, it is possible to integrate the judgment of different criteria directly. Hence principle of value-covered set of data of index-j, $(j=1, \cdots, n)$ is adopted.

Definition 2.7(Standard Grey Target of Assessment) Assume that $\otimes_{i j} \in\left[a_{i j}, b_{i j}\right],(j=1, \cdots, n)$ is a series assessments of number-n on fixed subject-i, which could be object-i, index-i, or source-i, and $\otimes_{i j}(j=1, \cdots, n)$ have the same measurement scale. Set standard Grey number, that is $\otimes_{S i}=\otimes_{\text {total }} \in\left[a_{s t}, b_{s t}\right], a_{s t} \neq b_{s t}, a_{s t}=\min _{j} a_{i j}, b_{s t}=\max _{j} b_{i j}$ $(j=1, \cdots, n) . \quad \otimes_{s i}$ is defined as Standard Grey Target of Assessment of $\otimes_{i j}(j=1, \cdots, n)$.

Definition 2.8(Standard Grey Potential Degree) To each judgment $\otimes_{i j} \in\left[a_{i j}, b_{i j}\right],(j=1, \cdots, n)$, set Standard Grey Target $\otimes_{s i}$ for fixed subject-i, Standard Grey Superiority Potential Degree of $\otimes_{i j}$ is, $G S P D_{s i \rightarrow i j}^{s}=\frac{b_{s t}-b_{i j}}{b_{s t}-a_{s t}}$; Standard Grey Inferior Potential Degree of $\otimes_{i j}$ is $\operatorname{GIPD}_{s i \rightarrow i j}^{S}=\frac{a_{s t}-a_{i j}}{b_{s t}-a s_{t}}$, Standard Grey Total Potential Degree of $\otimes_{i j}$ is $G T P D_{S i \rightarrow i j}^{S}=\alpha \cdot G S P D_{s i \rightarrow i j}^{S}+\beta \cdot G I P D_{s i \rightarrow i j}^{S}$, $\alpha+\beta=1, \alpha, \beta \in[0,1]$.

It is easy to proof that the natures of Standard Grey Potential Degree as followed, there are $0 \leq G S P D_{s i \rightarrow i j}^{S} \leq 1$, $-1 \leq G I P D_{s i \rightarrow i j}^{s} \leq 0$ and $-1 \leq G T P D_{s i \rightarrow i j}^{s} \leq 1$.

By the ways of Standard Grey Potential Degree, Grey target problem with Grey matrix could be deposed.

\section{GREY TARGET DECISION-MAKING MODELS}

In this paper the Basic Grey Target Clustering Model is discussed.

When a Grey target is provided, many subjects can be divided into different clusters which are in different relationship with Grey target. This kind of decision-making problem is called Grey target clustering.

Here the value of target and subjects are presented as Grey numbers. It is assumed that there is a series of evaluation $\otimes_{i} \in\left[a_{i}, b_{i}\right](i=1, \cdots, n)$ and target $\otimes_{T}$.

Above all, the realistic condition of target and each subject is complex because the relationship between two Grey numbers is so complex that $G S P D_{i \rightarrow j}$ and $G I P D_{i \rightarrow j}$ should be discussed separately and further combined to analyze.

Based on the conception and characters of Grey potential degree discussed above, combined with the Grey superiority and inferiority, the Grey potential relationship between Grey numbers $\otimes_{i}$ and $\otimes_{j}$ can be classified in to six kinds according to the Grey superiority potential degree $G S P D_{i \rightarrow j}$ and Grey inferiority potential degree $G I P D_{i \rightarrow j}$, as shown in Table I.

Grey potential degree of $\otimes_{i}$ to $\otimes_{j}$ is mainly composed of Grey superiority potential and Grey inferiority potential which present the relationship between Grey numbers $\otimes_{i}$ and $\otimes_{j}$. The case of "Relative Superiority and None Inferiority" is marked as the type of "RSNI" in abbreviated. And the case of "Relative Inferiority and None Superiority" is 
defined as the type of "RINS" in abbreviated. The conditions of "Relative Superiority and Relative Inferiority" and "None Superiority and None Inferiority" are similar and named as the type "RSRI" and "NSNI". Therefore by the Grey potential degree, there are six regions of Grey potential relationship, shown in Figure I.

In Figure I, the oval area is $\otimes_{j}$ and each kind of relationship has it typical Grey number. According to the relationship map of Grey numbers, for a series of evaluation $\otimes_{i} \in\left[a_{i}, b_{i}\right](i=1, \cdots, n)$ and target $\otimes_{T}$, the basic Grey target clustering is processed as follows.

TABLE I. THE GREY POTENTIAL RELATIONSHIP BETWEEN GREY NUMBERS $\otimes_{i}$ TO $\otimes_{j}$

\begin{tabular}{|c|c|c|c|}
\hline $\begin{array}{l}\begin{array}{l}\text { Grey } \\
\text { Potential } \\
\text { degree of }\end{array} \\
\otimes_{i} \quad \text { to } \\
\otimes_{j}\end{array}$ & $G S P D_{i \rightarrow j}$ & $G I P D_{i \rightarrow j}$ & $\begin{array}{c}\text { Mark } \\
\text { of } \\
\text { Relatio } \\
\text { nship }\end{array}$ \\
\hline $\begin{array}{l}\text { Absolutely } \\
\text { Superiority }\end{array}$ & $G S P D_{i \rightarrow j} \succ 1$ & & AS \\
\hline $\begin{array}{c}\text { Relative } \\
\text { Superiority } \\
\text { and None } \\
\text { Inferiority }\end{array}$ & $0 \leq G S P D_{i \rightarrow j} \leq 1$ & $G I P D_{i \rightarrow j} \succ 0$ & RSNI \\
\hline $\begin{array}{c}\text { Relative } \\
\text { Superiority } \\
\text { and } \\
\text { Relative } \\
\text { Inferiority }\end{array}$ & $0 \leq G S P D_{i \rightarrow j} \leq 1$ & $-1 \leq G I P D_{i \rightarrow j} \leq 0$ & $\begin{array}{l}\text { RSRI } \\
\text { RE1 }\end{array}$ \\
\hline $\begin{array}{c}\text { None } \\
\text { Superiority } \\
\text { and None } \\
\text { Inferiority }\end{array}$ & $G S P D_{i \rightarrow j} \prec 0$ & $G I P D_{i \rightarrow j} \succ 0$ & $\begin{array}{l}\text { NSNI } \\
\text { RE2 }\end{array}$ \\
\hline $\begin{array}{c}\text { Relative } \\
\text { Inferiority } \\
\text { and None } \\
\text { Superiority }\end{array}$ & $G S P D_{i \rightarrow j} \prec 0$ & $-1 \leq G I P D_{i \rightarrow j} \leq 0$ & NSRI \\
\hline
\end{tabular}

Step one. Computer the Grey superiority potential degree $G S P D_{i \rightarrow T}$ and Grey inferior potential degree $G I P D_{i \rightarrow T}$ separately.

Step two. According to Grey potential relationship between Grey numbers in Table I and make the relationship map of Grey target clustering.

This is the basic Grey target clustering method. In many situations, Grey target clustering is asked based on multiple criteria. It will be discussed in other papers.

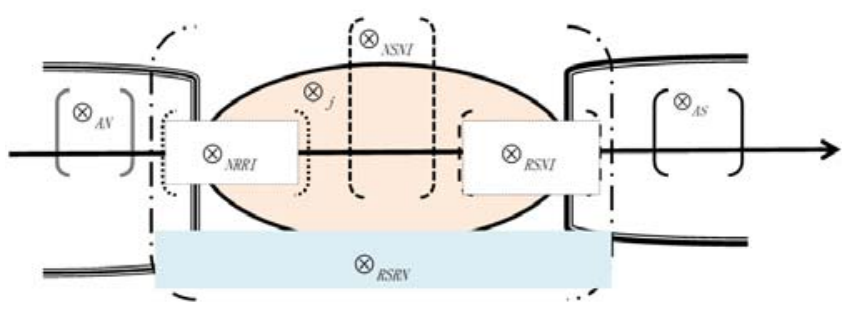

FIGURE I. THE RELATIONSHIP MAP BETWEEN GREY NUMBERS BY GREY POTENTIAL DEGREES

\section{EXAMPLES}

With the Grey Potential Degree and its application model in Section II and III, in this section one example of Grey Target Clustering will be presented to explain the effect of Grey potential-based method on Grey target decision.

When a Grey target $[80,85]$ is provided, there are 20 evaluations of subjects shown in Table II, which need to be divided into different clusters according to different relationship with Grey target.

There is a Grey target clustering problem. Here the value of target and subjects are presented as Grey numbers. There is a series of evaluation $\otimes_{i} \in\left[a_{i}, b_{i}\right](i=1, \cdots, 20)$ and target $\otimes_{T} \in[80,85]$.

TABLE II. TWENTY EVALUATIONS DATA OF SUBJECTS

\begin{tabular}{|c|c|c|c|}
\hline NO. & Score & NO. & Score \\
\hline 1 & {$[88,92]$} & 11 & {$[86,91]$} \\
\hline 2 & {$[80,85]$} & 12 & {$[78,85]$} \\
\hline 3 & {$[82,84]$} & 13 & {$[75,80]$} \\
\hline 4 & {$[83,88]$} & 14 & {$[63,68]$} \\
\hline 5 & {$[81,84]$} & 15 & {$[90,93]$} \\
\hline 6 & {$[82,88]$} & 16 & {$[80,84]$} \\
\hline 7 & {$[75,82]$} & 17 & {$[75,85]$} \\
\hline 8 & {$[78,86]$} & 18 & {$[75,87]$} \\
\hline 9 & {$[58,63]$} & 19 & {$[76,81]$} \\
\hline 10 & {$[85,90]$} & 20 & {$[80,88]$} \\
\hline
\end{tabular}

According to the model of Grey target clustering in Section III, the realistic condition of target and each subject depends on the relationship between Grey superiority potential degree $G S P D_{i \rightarrow T}$ and Grey inferior potential degree $G I P D_{i \rightarrow T}$.

Based on the conception and characters of Grey potential degree discussed above, combined with the Grey superiority and inferiority, the Grey potential relationship between Grey numbers $\otimes_{i}$ and $\otimes_{T}$ can be classified in to six kinds, as shown in Table III and IV.

TABLE III. $G S P D_{i \rightarrow T}, G I P D_{i \rightarrow T}$ AND GREY POTENTIAL RELATIONSHIP

\begin{tabular}{|c|c|c|c|}
\hline NO. & GSPD & GIPD & $\begin{array}{c}\text { Grey Potential } \\
\text { Relationship }\end{array}$ \\
\hline 1 & 1.750 & 2.000 & AS \\
\hline 2 & 0.000 & 0.000 & RSRI \\
\hline 3 & -0.500 & 1.000 & NSNI \\
\hline 4 & 0.600 & 0.600 & RSNI \\
\hline 5 & -0.333 & 0.333 & NSNI \\
\hline 6 & 0.500 & 0.333 & RSNI \\
\hline 7 & -0.429 & -0.714 & NSRI \\
\hline 8 & 0.125 & -0.250 & RSRI \\
\hline 9 & -4.400 & -4.400 & AI \\
\hline 10 & 1.000 & 1.000 & RSNI \\
\hline 11 & 1.2 & 1.2 & AS \\
\hline
\end{tabular}


The relevant research done in this paper is supported by

TABLE III, CONT. $G S P D_{i \rightarrow T}, G I P D_{i \rightarrow T}$ AND GREY POTENTIAL RELATIONSHIP

\begin{tabular}{|c|c|c|c|}
\hline 12 & 0.000 & -0.286 & RSRI \\
\hline 13 & -1.000 & -1.000 & NSRI \\
\hline 14 & -3.400 & -3.400 & AI \\
\hline 15 & 2.667 & 3.333 & AI \\
\hline 16 & -0.250 & 0.000 & NSRI \\
\hline 17 & 0.000 & -0.500 & NSRI \\
\hline 18 & 0.167 & -0.417 & RSRI \\
\hline 19 & -0.800 & -0.800 & NSRI \\
\hline 20 & 0.375 & 0.000 & RSRI \\
\hline
\end{tabular}

TABLE IV. SIX CLUSTERS OF TWENTY SUBJECTS

\begin{tabular}{|c|c|c|c|}
\hline $\begin{array}{c}\text { Grey } \\
\text { Potential } \\
\text { Relationship }\end{array}$ & NO. & $\begin{array}{c}\text { Grey } \\
\text { Potential } \\
\text { Relationship }\end{array}$ & NO. \\
\hline AI & $9,14,15$ & RSNI & $4,6,10$ \\
\hline AS & 1,11 & NSRI & $7,13,16,17,19$ \\
\hline NSNI & 3,5 & RSRI & $2,8,12,19,20$ \\
\hline
\end{tabular}

\section{CONCLUSIONS}

The description and treatment of uncertain information is very important to Grey target decision-making problem. This paper focuses on handling directly Grey number without whiten value and without distribution function in Grey target decision-making. The paper firstly built up the definition system of Grey potential degrees, the Grey Potential Degrees, including Grey Superiority Potential Degree, Grey Inferior Potential Degree, Grey Total Potential Degrees, and their characters are analyzed. In order to compare and sort different kinds of Grey numbers, standard Grey potential degree is put forward. Then the application of standard Grey potential degree, the model of Basic Grey Target Clustering Model is built up. Finally, three typical numerical example is presented to demonstrate the effectiveness of the method in Section IV.

Furthermore, approach of Grey potential degree could treat the data with both comparing and standardization work. Grey potential could be used as a form of metrics in Grey target decision-making in many situations of Grey target, including real number and interval number, whether single source or multiple sources.

As is presented in the results, the method proposed this paper can describe uncertain information better. The Grey potential-based method can makes full use of Grey number information without losing information and without additional information. And it can substitute stochastic and interval methods to solve uncertain problems in the situation that enough information of uncertainty is unavailable. It will be widely used in uncertain decision-making problems.

\section{ACKNOWLEDGMENT}

The authors sincerely express their heartiest thanks to Dr. Chaoqing Yuan and Xiaofeng Xu for their valuable comments and suggestions.
Zhejiang Provincial Natural Science Foundation Project (LY14G010005).

\section{REFERENCES}

[1] J.L. Deng, The control problem of Grey systems, Syst. Control Lett. 1 (1982) 288-294.

[2] Julong Deng, gray control system [J] Huazhong Institute of Technology [J]. 1982, 10(3): 9-18.

[3] Chanyao Gu and Wanhua Qiu, Entropy and Its Application in Bayes Decision [J]. Control and Decision, 1991, 6(4): 253-259.

[4] Mon D.L, Cheng C.H, Lin J.C. Evaluating Weapon System using Fuzzy Analytic Hierarchy Process based on Entropy Weight [J]. Fuzzy Sets and Systems, 1994, 62(2): 127-134.

[5] Wang Y.M, Elhag T.M.S.. A goal programming method for obtaining interval weights from an interval comparison matrix [J]. European Journal of Operational Research, 2007, 177(1):458-471.

[6] Sifeng Liu, Yaoguo Dang, Zhigeng Fang. Sifeng Liu [6], Yaoguo Dang. 2010:1-25.

[7] Yaoguo Dang,Sifeng Liu, Bin Liu. Grey Target Decision Model of Multiple Attribute Based on Interval Number [J]. China Engineering Science. 2005,7(8): 31-35.

[8] Yongming Chen, Haiying Xie. Statistical Simulation Test of the Incompatibility of Deng's Grey Target Transformation [J]. Systems Engineering and Electronics.2007, 29(8): 1285-1287.

[9] Guangzhi Bu, Yuwen Zhang. Grey Fuzzy Comprehensive Evaluation Based on Interval Numbers of Three Parameters [J]. Systems Engineering and Electronics.2001, 23(9): 43-45, 62.

[10] Qizhou Hu, Wei Zhang, Li Yu. Three Parameters Interval Numbers and Its Application in decision analysis [J]. China Engineering Science, 2007, 9(3): 47-51.

[11] Xinfeng Wang. Three parameters interval data aggregation operators and their application in decision making $[\mathrm{J}]$. Systems Engineering and Electronics. 2008, 30(8): 1468-1473.

[12] Rong Lan, Jiulun Fan. TOPSIS Decision Making in Three parameters interval value Fuzzy Sets [J]. Systems Engineering Theory and Practice, 2009,29(5): 129-136.

[13] Dang Luo. Decision Making of three - parameter interval Grey number [J]. Systems Engineering Theory and Practice. 2009, 29(1): 124-130.

[14] Jie Song. Grey Decision Making and application [D] Nanjing: Nanjing University of Aeronautics and Astronautics. 2010.

[15] Nai-ming Xie, Si-feng Liu, Novel methods on comparing Grey numbers, Applied Mathematical Modelling 34 (2010) 415-423

[16] Bo ZENG, Sifeng LIU, Chuan LI, Jiumei CHEN, Grey target decisionmaking model of interval Grey number based on cobweb area, Systems Engineering and Electronics, Vo1. 35 No. 11, November 2013, 23292334

[17] Fang Zhigeng, Research on the Game Theory and its Application in Economy, A Thesis for the Degree of Doctor of Philosophy, Nanjing University of Aeronautics and Astronautics, September, 2007, 120 -123.

[18] Aiqing Ruan, Zhigeng Fang, Grey Potential Degrees and its Application in Grey Game, The Journal of Grey System, Vol.25 No.2,2013

[19] Ruan Aiqing and Wang Yinao, The Further Discussion of Distribution and Characters of Grey Potential Degrees The Journal of Grey System, Vol.26 No.4,2014. 